\title{
Stichwort: Der Mythos der nordischen Bildungssysteme
}

\author{
Mareike Kobarg • Manfred Prenzel
}

\begin{abstract}
Zusammenfassung: Dieser Beitrag widmet sich der Frage, ob die Bildungsergebnisse, die nordische Staaten in internationalen Schulleistungsuntersuchungen erzielen, den Mythos gerechter und leistungsstarker nordischer Bildungssysteme, der in Deutschland häufig diskutiert wird, rechtfertigen. Um diese Frage zu beantworten, werden Befunde aus den vergangenen drei Runden des Programme for International Student Assessment (PISA) für die nordischen Länder vorgestellt. Die Ergebnisse zeigen, dass keineswegs alle nordischen Staaten über alle Domänen hinweg herausragende Leistungen erzielen. Allein Finnland erreicht im internationalen Vergleich ausnahmslos die Spitzengruppe. Gleichzeitig finden sich Hinweise darauf, dass die nordischen Staaten größtenteils erfolgreich mit den sozialen Disparitäten umgehen, die Schülerinnen und Schüler in die Schule mitbringen. Betrachtet man Bedingungen auf der Ebene von Schule und Unterricht, so weichen die nordischen Staaten jedoch nur geringfügig vom OECD-Durchschnitt ab. Insgesamt scheint eine idealisierende Betrachtung aller nordischen Bildungssysteme aufgrund der Bildungsergebnisse, die sie in der internationalen Schulleistungsuntersuchung PISA erzielt haben, kaum gerechtfertigt.
\end{abstract}

Schlüsselwörter: PISA · Nordische Staaten · Kompetenzen · soziale Disparitäten · Schule · Unterricht

\section{Keyword: The myth of the Nordic educational systems}

\begin{abstract}
This paper deals with the question of whether the educational outcomes of the Nordic countries displayed in international comparative studies justify the legendary-status of fair and high-performing Nordic educational systems which is often discussed in Germany. To answer this question, the results of the Nordic countries in the past three surveys of the "Programme for International Student Assessment" (PISA) are presented. The results show that not all of the Nordic countries reach outstanding results in all of the investigated domains. It is only Finland that is always, without exception, found at the top of the international comparison. At the same
\end{abstract}

\footnotetext{
Online publiziert: 14.01 .2010

(C) Die Autoren 2010. Dieser Artikel ist auf Springerlink.com mit Open Access verfügbar.

Dr. M. Kobarg ( $\bowtie)$

IPN - Leibniz-Institut für die Pädagogik der Naturwissenschaften und Mathematik an der Universität Kiel, Olshausenstr. 62, 24098 Kiel, Deutschland

E-Mail: kobarg@ipn.uni-kiel.de

Univ.-Prof. M. Prenzel

TU München, TUM School of Education, Schellingstr. 33, 80799 München, Deutschland E-Mail: manfred.prenzel@tum.de
} 
time, the findings point out that the Nordic countries deal with the social disparities that students bring to school successfully. When considering contextual factors on a school and classroom level, however, the Nordic countries only differ slightly from the OECD average. To conclude, on the basis of the results achieved in the international comparative study PISA, an idealisation of the Nordic educational systems hardly seems justified.

Keywords: PISA $\cdot$ Nordic countries $\cdot$ Competencies $\cdot$ Social disparities $\cdot$ School $\cdot$ Instruction

\section{Einleitung}

Für eine kleine Gruppe von Touristen galt der Norden schon immer als Geheimtipp. Mit der Veröffentlichung der ersten PISA-Ergebnisse (Baumert et al. 2001b; OECD 2001) zog es, unabhängig von der Reisesaison, eine andere Gruppe in die nördlichen Staaten Europas: Bildungspolitiker und -politikerinnen, Vertreterinnen und Vertreter von Lehrerverbänden, Journalisten und Journalistinnen sowie Fachleute für Unterricht und Schule. Finnland war die Überraschung der ersten Runde des „Programme for International Student Assessment" (PISA). Schweden oder auch Island arrondierten mit sehr guten Ergebnissen den Eindruck des bildungsstarken Nordens.

Zum schlagartig einsetzenden Interesse an den Bildungssystemen der nordischen Staaten könnte auch der Übergang von TIMSS (Third International Mathematics and Science Study) zu PISA beigetragen haben. In Deutschland hatten bereits die TIMSS-Befunde (Baumert et al. 1997; Beaton et al. 1996) Aufmerksamkeit gefunden und einen ,Schock“ ausgelöst. Bei dieser auf Mathematik und Naturwissenschaften fokussierten Vergleichsstudie zeigten asiatische Staaten (insbesondere Japan) Leistungsergebnisse, von denen Deutschland nur träumen konnte. Die Vergleichsdistanz über die Kulturräume war allerdings beträchtlich. Schweden war bei TIMSS zwar durchaus erfolgreich und insbesondere in den Darstellungen der Bildungssysteme gewürdigt worden (Robitaille 1997); doch Finnland hatte auf eine Teilnahme an TIMSS sowie an weiteren Studien der ,International Association for the Evaluation of Educational Achievement" (IEA) verzichtet.

Im härteren Vergleich der OECD-Staaten, der nun auch das Leseverständnis einbezog, erschienen die in verschiedener Hinsicht näher an Deutschland liegenden nördlichen Länder als sehr viel kritischere Vergleichsgruppe. Das Image der nordischen Staaten stand auch nicht im Verdacht für angepasstes oder stures Lernen.

Die in den Medien Deutschlands präsentierten Reiseberichte verstärkten das Interesse an den Bildungssystemen der nordischen Staaten. Aber auch eher nüchterne Vergleiche von erfolgreichen Bildungssystemen (Bundesministerium für Bildung und Forschung 2003) bekräftigten den Eindruck, dass man im Norden Europas die Perspektiven für die Schule der Zukunft finden könnte: Erfolgreiches gemeinsames Lernen bis zum Ende der Sekundarstufe, keine Ausgliederung von Schülerinnen und Schülern an Sonder- oder Förderschulen, spät einsetzende Zensuren, aber dennoch ausgezeichnete Kompetenzen bei einer geringen Leistungsstreuung und relativ schwachen Zusammenhängen mit sozialer Herkunft (vgl. auch OECD 2001, 2004, 2007a). Wissenschaftlerinnen und Wissenschaftler aus nordischen Staaten skizzierten geteilte Grundüberzeugungen wie eine hohe Wertschätzung von Bildung, Chancengerechtigkeit und individueller Förderung (Hautamäki et al. 2008; Lie et al. 2003). Solche Darstellungen wurden und werden ebenso gerne auf- 
gegriffen wie begeisterte Berichte von Austauschlehrkäften. Große Kreise pädagogisch Interessierter goutierten die ausführlichen und einfühlsamen Mediendokumentationen (z. B. Kahl 2003), die Besonderheiten der Schulen in Finnland und Schweden anschaulich werden ließen. Bis heute wird solchen Berichten (z. B. Sarjala u. Häkli 2008) aus dem Norden in Deutschland große Aufmerksamkeit zuteil.

Obwohl die Ähnlichkeiten der nordischen Bildungssysteme häufig hervorgehoben werden, findet man - bei genauer Betrachtung - auch nennenswerte Unterschiede zwischen ihnen. So gibt es zum Beispiel in Dänemark ein dezentral angelegtes Bildungssystem mit viel Entscheidungsspielraum für einzelne Schulen, wohingegen die Schulen in Norwegen stark zentral gesteuert werden. Auch Schweden und Finnland zeichneten sich lange durch ein zentralisiertes Schulsystem aus. In den letzten 20 Jahren gibt es in diesen Staaten jedoch eine starke Tendenz zur Dezentralisierung. Weitere Unterschiede bestehen etwa in den Anteilen und der Rolle von Privatschulen in den nordischen Bildungssystemen, die nur in Dänemark und Schweden von knapp zehn Prozent der Schülerinnen und Schüler besucht werden; in den anderen nordischen Staaten beträgt ihr Anteil weniger als fünf Prozent (Matti 2009). Weitere Unterschiede betreffen beispielsweise die Entscheidungsspielräume der Eltern bei der Wahl der Schule, die Art der (einheitlichen) Abschlussprüfungen und die Notenstufen.

Die Darstellungen nordischer Bildungssysteme und ihrer Erfolge haben sich möglicherweise bereits zu einer Idealvorstellung einer optimalen Lehr-Lern-Landschaft verfestigt, die auf allen Ebenen des Bildungssystems vorbildlich ist und somit als realistischer Bezugspunkt für die Weiterentwicklung des Schulsystems in Deutschland dienen kann. Sicher kann eine Funktion von internationalen Vergleichen durchaus darin gesehen werden, konkrete Visionen oder Benchmarks für die Weiterentwicklung von Bildungssystemen anzubieten (Seidel u. Prenzel 2008). Ist aber eine Idealisierung des nordischen Bildungssystems, das in Finnland und Skandinavien in den Grundzügen gleich angelegt ist, wirklich gerechtfertigt? Gelingt es diesen Staaten gleichermaßen, einer großen Zahl ihrer Schülerinnen und Schüler die Kompetenzen zu vermitteln, die sie für das Leben und Weiterlernen in einer Wissensgesellschaft benötigen? Garantiert die Anlage der Bildungssysteme in den nordischen Staaten stabile Lernerfolge über längere Zeiträume und mit Blick auf die Zukunft? - Mit diesen Fragen befasst sich der vorliegende Überblicksartikel. Grundlage sind Daten aus der internationalen Schulleistungsuntersuchung PISA, die seit dem Jahr 2000 alle drei Jahre durchgeführt wird (OECD 2001, 2004, 2007a). PISA untersucht die Kompetenzen von fünfzehnjährigen Jugendlichen (in vielen Staaten also am Ende der regulären Schullaufbahn), die sowohl für individuelle Lern- und Lebenschancen als auch für die gesellschaftliche, politische und wirtschaftliche Weiterentwicklung bedeutsam sind. Hierbei konzentrieren sich die Erhebungen auf die drei Domänen Lesen, Mathematik und Naturwissenschaften (Prenzel et al. 2007a). Neben den Kompetenzen der Jugendlichen werden auch eine Reihe von Kontextfaktoren auf der Ebene der Länder, Schulen und der Individuen erfasst (Baumert et al. 2001a; Prenzel 2005), um Zusammenhänge zwischen ihnen und den Bildungsergebnissen von Schülerinnen und Schülern zu beschreiben. Damit werden in PISA Daten erfasst, mit deren Hilfe die Frage nach dem Erfolg der nordischen Bildungssysteme im Hinblick auf einige entscheidende Indikatoren beleuchtet werden kann. 
Da offensichtlich die erste PISA Datenerhebung im Jahr 2000 (OECD 2001) den Mythos der erfolgreichen Bildungssysteme der nordischen Staaten begründete, sollen die Befunde der nordischen Staaten in dieser Erhebung genauer betrachtet und dann auf die Ergebnisse der nachfolgenden Erhebungsrunden bezogen werden. Anschließend wenden wir uns der Frage zu, wie unterschiedliche Kontextfaktoren in den nordischen Ländern mit den Bildungsergebnissen von Schülerinnen und Schülern zusammenhängen. Auch diese Analysen können Hinweise darauf liefern, wie erfolgreich die nordischen Bildungssysteme insbesondere auch im Hinblick auf eines ihrer wichtigsten Ziele - die Chancengerechtigkeit - tatsächlich sind.

\section{Wie erfolgreich sind die nordischen Bildungssysteme? Befunde aus drei PISA-Runden}

Anhand der Ergebnisse bei PISA kann untersucht werden, inwieweit die nordischen Staaten und Bildungssysteme Schülerinnen und Schüler insgesamt und in ähnlicher Weise in der Entwicklung relevanter Kompetenzen unterstützen. Wir berichten zunächst die Befunde für PISA 2000 und betrachten dann die Veränderungen über die bisherigen PISA-Erhebungen (2000, 2003, 2006).

\subsection{Kompetenzen der Jugendlichen in den nordischen Staaten bei PISA 2000}

Das Interesse für das Bildungssystem in Finnland und den anderen nordischen Staaten wurde durch die Ergebnisse der ersten PISA-Erhebung im Jahr 2000 (OECD 2001) geweckt. In Tab. 1 sind die Kompetenzmittelwerte und Standardabweichungen für die nordischen Staaten bei PISA 2000 dargestellt (vgl. auch Baumert et al. 2001b). Als Bezugspunkte werden auch die OECD-Kennwerte und die Ergebnisse der deutschen Schülerinnen und Schüler aufgelistet.

Tab. 1: Mittelwerte und Standardabweichungen der Kompetenzwerte in den Domänen Lesen, Mathematik und Naturwissenschaften für die nordischen Länder und Deutschland bei PISA 2000 (vgl. OECD 2001, S. 253, 259, 261)

\begin{tabular}{|c|c|c|c|c|c|c|c|c|c|}
\hline \multirow[b]{2}{*}{ Staat } & \multicolumn{3}{|c|}{ Lesen } & \multicolumn{3}{|c|}{ Mathematik } & \multicolumn{3}{|c|}{ Naturwissenschaften } \\
\hline & $\bar{M}$ & $(S E)$ & $\overline{S D}$ & $\bar{M}$ & $(S E)$ & $\overline{S D}$ & $\bar{M}$ & $(S E)$ & $S D$ \\
\hline Dänemark & 497 & $(2,4)$ & 98 & 514 & $(2,4)$ & 87 & 481 & $(2,8)$ & 103 \\
\hline Finnland & 546 & $(2,6)$ & 89 & 536 & $(2,2)$ & 80 & 538 & $(2,5)$ & 86 \\
\hline Island & 507 & $(1,5)$ & 92 & 514 & $(2,3)$ & 85 & 496 & $(2,2)$ & 88 \\
\hline Norwegen & 505 & $(2,8)$ & 104 & 499 & $(2,8)$ & 92 & 500 & $(2,8)$ & 96 \\
\hline Schweden & 516 & $(2,2)$ & 92 & 510 & $(2,5)$ & 93 & 512 & $(2,5)$ & 93 \\
\hline Deutschland & 484 & $(2,5)$ & 111 & 490 & $(2,5)$ & 103 & 487 & $(2,4)$ & 102 \\
\hline OECD-Durchschnitt & 500 & $(0,6)$ & 100 & 500 & $(0,7)$ & 100 & 500 & $(0,7)$ & 100 \\
\hline
\end{tabular}

Fettgedruckte Werte liegen signifikant über dem OECD-Mittelwert

Kursivgedruckte Werte liegen signifikant unter dem OECD-Mittelwert 
Die in Tab. 1 zusammengefassten Befunde zeigen, dass alle nordischen Staaten in den drei Kompetenzbereichen - mit Ausnahme der naturwissenschaftlichen Kompetenz in Dänemark - (signifikant) besser abschneiden als Deutschland, das durchgehend signifikant unter dem OECD-Mittelwert liegt. Dieses Bild einer weitreichenden Überlegenheit in den Leistungsvergleichen könnte aus deutscher Perspektive tatsächlich die Basis für einen Mythos bilden.

Ein stärker fokussierter Blick auf die Befunde offenbart jedoch, dass letztlich nur zwei der fünf nordischen Länder - nämlich Finnland und Schweden - bei PISA 2000 in allen drei Domänen Kompetenzwerte erzielten, die signifikant über dem OECD-Durchschnitt liegen. In den drei anderen nordischen Ländern sind die Befunde insgesamt weniger beeindruckend. Die Kompetenzwerte liegen in diesen Staaten nur vereinzelt signifikant oberhalb des OECD-Durchschnitts, beispielsweise die Lesekompetenz der isländischen Jugendlichen oder die mathematische Kompetenz der Fünfzehnjährigen in Island und Dänemark. Besondere Beachtung verdienen die Leistungsstreuungen in den nordischen Ländern und hier besonders in Finnland und Schweden, die deutlich geringer ausfallen als im Durchschnitt der OECD. Diese Befunde widersprechen der damals in Deutschland weit verbreiteten Auffassung, dass man eine hohe Leistungsstreuung in Kauf nehmen muss, wenn man ein im Mittel sehr hohes Leistungsniveau erreichen will. Finnland und Schweden sind Beispiele für die pädagogische Wunschvorstellung, hohe und zugleich wenig heterogene Leistungen zu erreichen. Dieses Ergebnis war tatsächlich von einer besonderen Qualität.

Obwohl das Abschneiden der nordischen Länder bei PISA 2000 beeindrucken kann, bleiben Zweifel, ob die nordischen Staaten insgesamt als Spitzengruppe bezeichnet werden können. Zwei der nordischen Länder, namentlich Finnland und Schweden, liegen in allen Domänen oberhalb des OECD-Durchschnitts, wobei nur Finnland der absoluten Spitze im Vergleich aller OECD-Staaten zuzurechnen ist. Die drei weiteren nordischen Staaten sind hingegen eher im Mittelfeld der Rangreihe platziert. Somit weisen die Befunde der ersten PISA-Erhebung nicht generell darauf hin, dass die nordischen Staaten im gleichen Maße erfolgreich sind. Trotz der großen Ähnlichkeit ihrer Bildungssysteme und ihrer Werthaltung gegenüber Bildung variieren ihre Bildungsergebnisse in einer Größenordnung von bis zu 50 Punkten auf der PISA-Skala (z. B. zwischen Finnland und Dänemark im Lesen und in den Naturwissenschaften). Es sind also insbesondere die Schülerinnen und Schüler in Finnland, die sich im internationalen Vergleich auszeichnen, sich zugleich aber von den Jugendlichen in den anderen nordischen Staaten unterscheiden.

\subsection{Veränderung der Kompetenzen in den nordischen Staaten von PISA 2000 bis PISA 2006}

Es war die Absicht der OECD, PISA so anzulegen, dass Vergleiche über die Erhebungen im dreijährigen Abstand angestellt werden können (vgl. Carstensen et al. 2008). Damit kann auch beurteilt werden, ob das in einer Runde beobachtete Kompetenzniveau in den Staaten stabil bleibt, sich verbessert oder verschlechtert. Inwieweit erweisen sich also die Kompetenzergebnisse in den nordischen Staaten als stabil über die Zeit? Stabil positive oder gar steigende Kompetenzwerte wären tatsächlich ein Grund, die nordischen Staaten mit ihren Bildungssystemen als Ideal für andere Länder zu betrachten. 


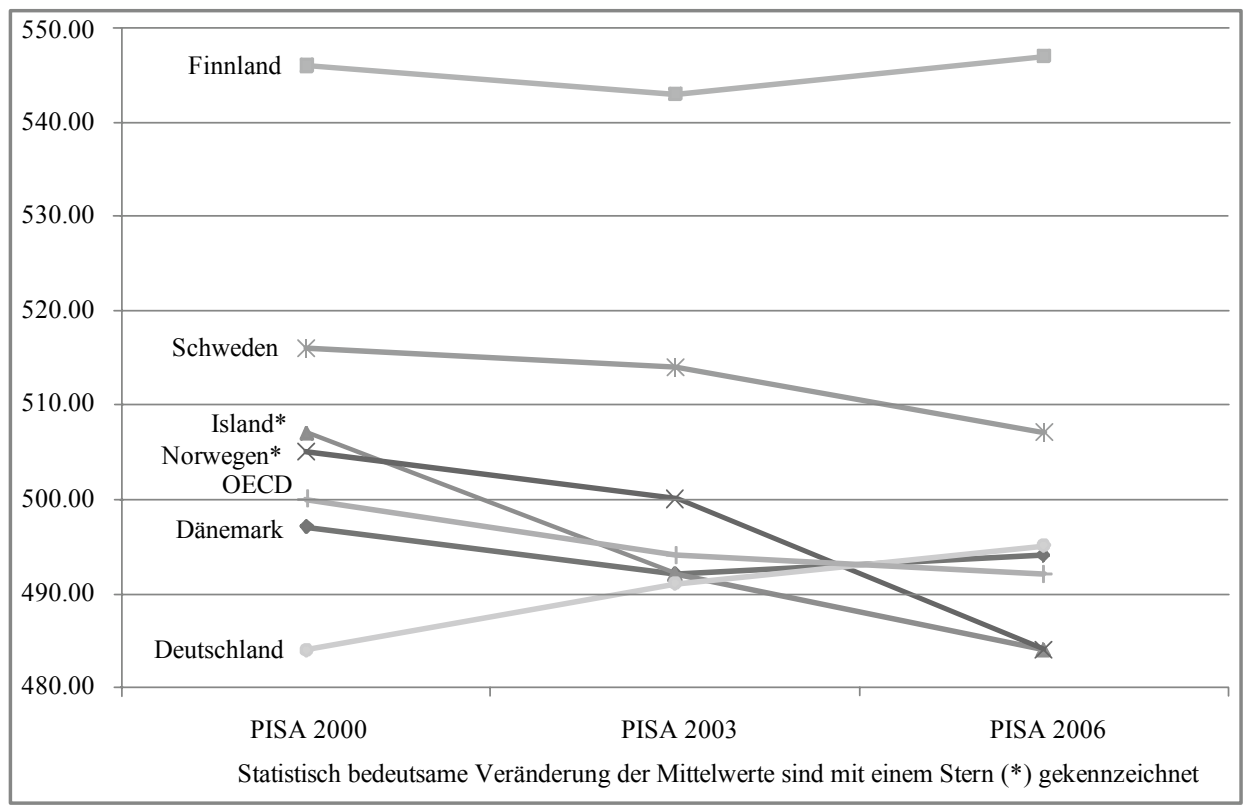

Abb. 1: Veränderung der Lesekompetenz in den nordischen Ländern, in Deutschland und im OECD-Durchschnitt von PISA 2000 bis PISA 2006 (vgl. Drechsel u. Artelt 2007, S. 412)

Für den Bereich der Lesekompetenz besteht eine ausreichend große Datenbasis, um die Veränderungen über alle drei Erhebungszeitpunkte hinweg zu berichten (Carstensen et al. 2008; Carstensen et al. 2007). Diese Veränderungen sind in Abb. 1 für die nordischen Länder, für Deutschland und im OECD-Durchschnitt dargestellt (vgl. Drechsel u. Artelt 2007).

Die Abb. 1 lässt sehr schön erkennen, dass Finnland in der Lesekompetenz - nicht nur im Vergleich zu Deutschland und zum OECD-Mittel, sondern auch zu den anderen nordischen Staaten - auf einem deutlich höheren Niveau liegt. Gleichzeitig zeigt die Abbildung, dass der Erfolg Finnlands über alle drei Erhebungszeitpunkte stabil bleibt.

In Norwegen und Island hingegen hat sich die Lesekompetenz der Fünfzehnjährigen über die drei Erhebungszeitpunkte statistisch bedeutsam verschlechtert. Ein massiver Abwärtstrend ist insbesondere in Island zu beobachten: Dort erreichten die Schülerinnen und Schüler bei PISA 2000 im Lesen noch signifikant bessere Kompetenzwerte als der OECD-Durchschnitt, aber auch als Deutschland. Bei PISA 2006 liegen die Kompetenzwerte der isländischen Fünfzehnjährigen nun deutlich unter dem OECD-Durchschnitt und auch unter dem Wert der deutschen Schülerinnen und Schüler.

Die tendenziellen Zunahmen in Deutschland können ebenso wenig statistisch abgesichert werden, wie die tendenziellen Abnahmen in Schweden und in Dänemark. Zusammen mit den signifikanten Abnahmen in Island und Norwegen lässt sich für die Gruppe der nordischen Staaten (mit Ausnahme von Finnland) keine Stabilität auf einem hohen Niveau der Lesekompetenz feststellen. Nur in Finnland finden wir für die Lesekompetenz tatsächlich stabile Werte. 


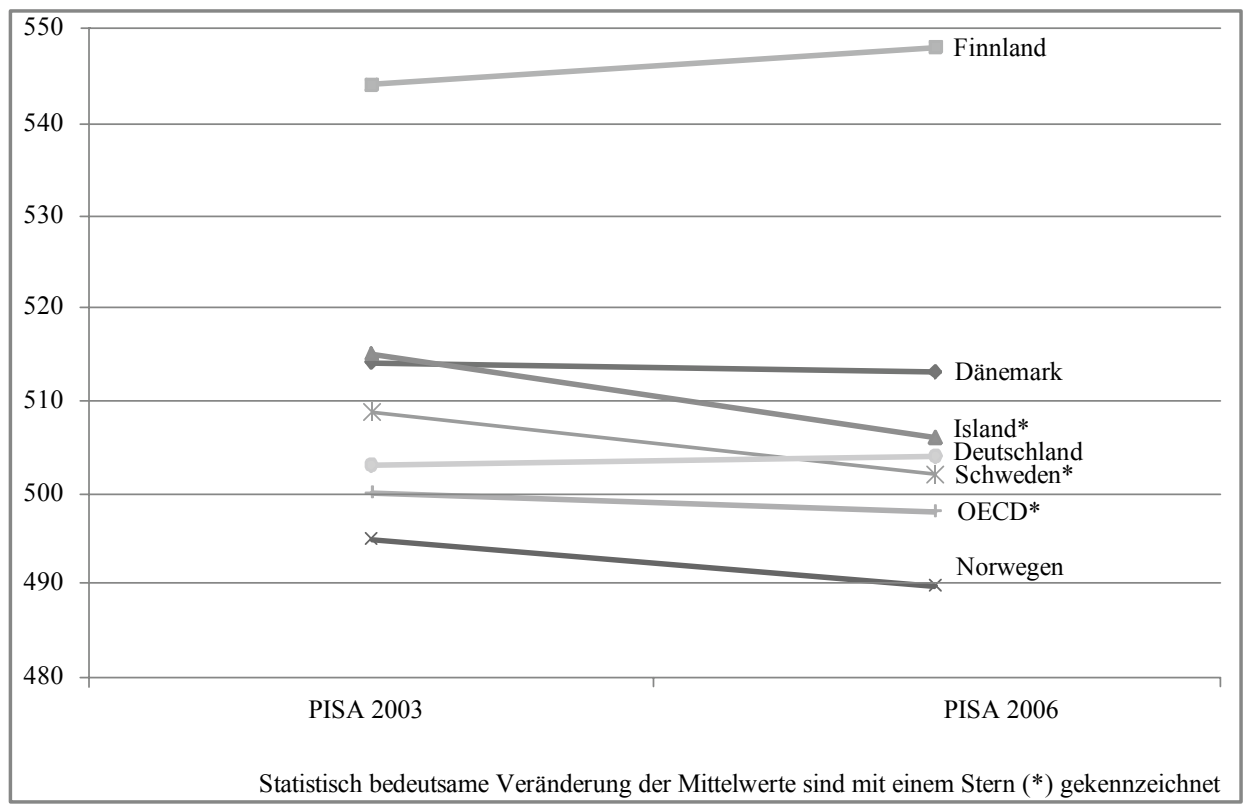

Abb. 2: Veränderung der mathematischen Kompetenz auf der Gesamtskala in den nordischen Ländern und in Deutschland von PISA 2003 bis PISA 2006 (vgl. Frey et al. 2007, S. 265)

Die Veränderungen der mathematischen Kompetenz lassen sich nicht über alle drei Erhebungszyklen abbilden, da hierzu die Menge und Zusammensetzung der verwendeten Aufgaben nicht ausreicht (Carstensen et al. 2007; Carstensen et al. 2008). Deswegen wird im Folgenden nur die Entwicklung auf der Mathematikgesamtskala zwischen PISA 2003 und PISA 2006 betrachtet. Abbildung 2 stellt diese Veränderung in den nordischen Ländern, in Deutschland und im OECD-Durchschnitt graphisch dar.

Ähnlich wie für die Lesekompetenz sind auch für die mathematische Kompetenz stabile Werte für Finnland - auf gleichbleibend hohem Niveau - aber auch für Dänemark und Deutschland auf der Gesamtskala von 2003 zu 2006 zu verzeichnen. In Norwegen sind die Unterschiede zwischen 2003 und 2006 nicht statistisch bedeutsam. In zwei der nordischen Länder - Island und Schweden - wird hingegen eine deutliche Abnahme der mathematischen Kompetenz zwischen PISA 2003 und PISA 2006 und damit eine negative Veränderung beobachtet.

Die Entwicklung der naturwissenschaftlichen Kompetenz lässt sich ausschließlich zwischen PISA 2000 und PISA 2003 über alle Staaten reliabel beschreiben (Carstensen et al. 2007; Carstensen et al. 2008). In Abb. 3 sind diese Entwicklungen in den nordischen Staaten, im OECD-Durchschnitt und in Deutschland dargestellt.

Die dargestellten Befunde zeigen, dass die durchschnittliche naturwissenschaftliche Kompetenz der finnischen Schülerinnen und Schüler sich zwischen PISA 2000 und PISA 2003 bedeutsam verbessert hat und damit stabil oberhalb des OECD-Durchschnitts und auch der deutschen Ergebnisse liegt. Doch auch in Deutschland ist ein bedeutsamer Anstieg der naturwissenschaftlichen Kompetenz zu verzeichnen. Eine negative Ent- 


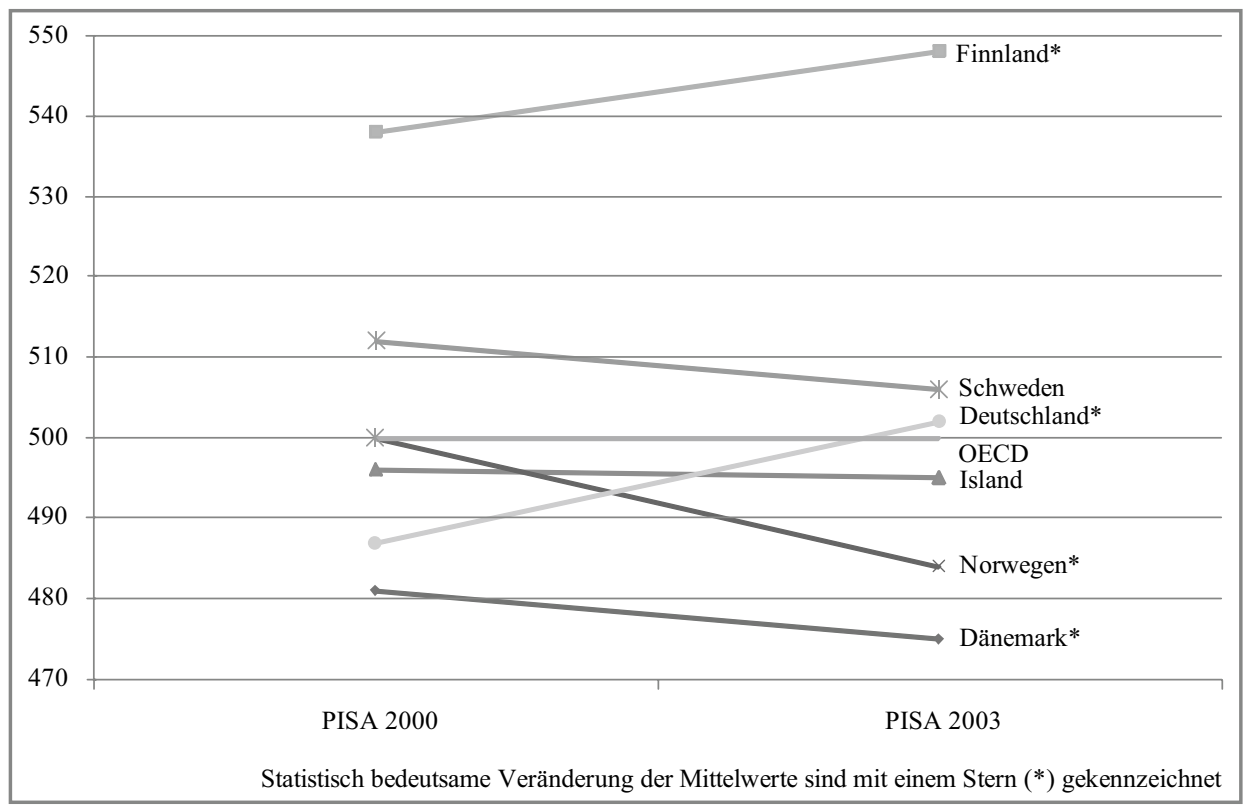

Abb. 3: Veränderung der naturwissenschaftlichen Kompetenz in den nordischen Ländern und in Deutschland von PISA 2000 bis PISA 2003 (vgl. Rost et al. 2004, S. 121)

wicklung der naturwissenschaftlichen Kompetenz zeigt sich hingegen in Norwegen und Dänemark, die bei PISA 2003 Kompetenzwerte erreichen, die deutlich unter dem OECDDurchschnitt und auch unter dem deutschen Wert liegen. Nur in Island und Schweden weisen die Fünfzehnjährigen bei PISA 2000 und bei PISA 2003 ähnliche Kompetenzwerte in den Naturwissenschaften auf.

Der Blick auf die Veränderung der Kompetenzen in den drei Domänen zeigt, dass die Ergebnisse keineswegs in allen nordischen Ländern stabil und auf einem hohen Niveau bleiben. Einzig in Finnland sind die Kompetenzwerte in allen drei Domänen über die drei Erhebungszeitpunkte hinweg stabil oder werden sogar besser (z. B. in den Naturwissenschaften). In allen anderen nordischen Ländern treten in einer oder mehreren Domänen negative Veränderungen der durchschnittlichen Kompetenz über die drei Erhebungszeitpunkte hinweg auf. In Deutschland hingegen verändern sich die Kompetenzen tendenziell positiv.

\subsection{Fazit - Sind die nordischen Bildungssysteme im internationalen Vergleich} besonders erfolgreich?

Die Befunde aus den drei PISA-Erhebungen in den Jahren 2000, 2003 und 2006 zeigen, dass die nordischen Länder im internationalen Vergleich keineswegs durchgängig Spitzenpositionen einnehmen, die eine Idealisierung der nordischen Bildungssysteme rechtfertigen könnten. Das Bild, das die PISA-Erhebungen zeichnen, ist deutlich differenzierter. Im internationalen Vergleich schneiden allein die finnischen Fünfzehnjährigen konstant 
herausragend ab. Finnland erreicht in allen Domänen Spitzenplätze und die Kompetenzen der finnischen Schülerinnen und Schüler sind auch über die drei Erhebungszeitpunkte stabil. In keinem anderen nordischen Land ist der Erfolg des Bildungssystems so konstant wie in Finnland. Obwohl Schweden in der ersten PISA-Erhebung im Jahr 2000 bedeutsam oberhalb des OECD-Durchschnitts lag, haben sich die Kompetenzen der schwedischen Fünfzehnjährigen tendenziell und in einigen Bereichen signifikant negativ entwickelt. Doch auch in anderen nordischen Staaten zeichnen sich Stagnationen oder Abnahmen in den Kompetenzwerten über die PISA-Erhebungen ab. Ein Grund hierfür liegt eventuell in den fortlaufenden Reformen, die besonders im schwedischen, aber auch in anderen nordischen Bildungssystemen seit den 1990er-Jahren vorangetrieben wurden und die ihre Wirkung im Verlauf der drei PISA-Erhebungszyklen entfaltet haben (Matti 2009). Damit liefern diese Befunde jedoch keine Belege dafür, dass eine ähnliche Bildungslandschaft mit einer hohen Wertschätzung für Bildung - wie sie in den nordischen Staaten zu finden ist - zwangsläufig zu erfolgreichen Bildungsergebnissen führt. Sie weisen aber auch darauf hin, dass Reformen im Bildungswesen sich auf unterschiedliche Art und Weise auf die Bildungsergebnisse eines Landes auswirken können.

\section{Bedingungsfaktoren für Bildungsergebnisse in den nordischen Staaten bei PISA}

Bisher haben wir gezeigt, dass die Kompetenzen der Jugendlichen in den nordischen Ländern zwar relativ konstant den OECD-Durchschnitt erreichen, aber nicht alle nordischen Länder der Spitzengruppe zuzuordnen sind. Im Anschluss an die Darstellung der Bildungsergebnisse der nordischen Länder stellt sich nun die Frage nach Bedingungsfaktoren in den nordischen Bildungssystemen und ihren Zusammenhängen mit diesen Bildungsergebnissen. Im Folgenden werden deshalb der Umgang mit sozialer Disparität sowie Merkmale von Unterricht und Schule in den nordischen Staaten diskutiert.

\subsection{Soziale Disparitäten}

Schülerinnen und Schüler sollen in den Bildungssystemen demokratischer Staaten unabhängig von ihrer sozialen, ethnischen und kulturellen Herkunft und von ihrem Geschlecht gleiche Chancen haben, Kompetenzen zu entwickeln, die für die gesellschaftliche Teilhabe relevant sind (Aktionsrat Bildung 2007; Lie et al. 2003). Wie gut es in den nordischen Staaten - auch im Vergleich zu Deutschland - gelingt, diesen Anspruch umzusetzen, d. h. soziale Disparitäten auszugleichen, soll im Folgenden untersucht werden.

\subsubsection{Soziale Herkunft}

Die soziale Herkunft ist ein Merkmal, das im Rahmen der internationalen PISA-Erhebungen durch unterschiedliche Aspekte erfasst wird, die entweder als einzelne Indikatoren oder als zusammengefasster Index in die weiteren Analysen mit einbezogen werden. In den von der OECD publizierten Analysen wird als Kennwert für die soziale Herkunft der sozio-ökonomische und -kulturelle Status, der ESCS (Economic, Social and Cultural 
Status) verwendet. Dieser Index berücksichtigt gleichzeitig ökonomische, kulturelle und soziale Herkunftsmerkmale (Ehmke u. Baumert 2007; Ehmke u. Siegle 2005).

Betrachtet man den ESCS als Kennwert für die soziale Herkunft, dann liegen die Mittelwerte der nordischen Staaten - wie auch in Deutschland - deutlich oberhalb des OECD-Mittelwerts (OECD 2007b, S. 124). Dies gilt auch für den gebräuchlichen Kennwert des (höchsten) sozioökonomischen Status in der Familie (HISEI), der bei den detaillierten Analysen in Deutschland vorgezogen wurde, da dieser Index robust ist und nicht (wie der ESCS von der OECD) über die Erhebungsrunden neu definiert wurde (Ehmke u. Baumert 2007).

Neben der Höhe des sozioökonomischen Status ist im internationalen Vergleich auch der Zusammenhang zwischen dem sozioökonomischen Hintergrund und der Kompetenz der Jugendlichen von Interesse, da er anzeigt, inwieweit es im Bildungssystem gelingt, soziale Disparitäten aufzufangen. Tabelle 2 stellt die sozialen Gradienten der Lesekompetenz in den nordischen Ländern, in Deutschland und im OECD-Durchschnitt bei PISA 2000, 2003 und 2006 dar. In Tab. 2 sind die Steigung des sozialen Gradienten $(b)$ und der Anteil der durch den sozioökonomischen Status aufgeklärten Varianz $\left(R^{2}\right)$ angegeben.

Der Blick auf Tab. 2 zeigt, dass der soziale Gradient der Lesekompetenz in allen nordischen Ländern in allen drei Erhebungsrunden im Bereich des OECD-Durchschnitts oder darunter liegt. Insbesondere in Finnland und Island ist der soziale Gradient der Lesekompetenz vergleichsweise klein. In Deutschland hingegen ist der Zusammenhang zwischen der sozialen Herkunft (HISEI) und der Lesekompetenz in allen Erhebungsrunden deutlich höher als im OECD-Durchschnitt.

Im Durchschnitt aller OECD-Staaten ist der soziale Gradient der Lesekompetenz über die drei Erhebungsrunden hinweg weitgehend gleich geblieben. Ähnliches gilt für die Mehrzahl der nordischen Länder. Nur in Dänemark und Deutschland hat die Steigung des sozialen Gradienten zwischen PISA 2000 und PISA 2006 statistisch bedeutsam abgenommen (Ehmke u. Baumert 2007; OECD 2007b).

In den nordischen Staaten ist der sozioökonomische Status von Jugendlichen - ähnlich wie in Deutschland - im Mittel vergleichsweise gut. Im Hinblick auf die Kopplung der

Tab. 2: Vergleich der sozialen Gradienten (HISEI) der Lesekompetenz bei PISA 2000, 2003 und 2006 für die nordischen Länder und Deutschland (vgl. Ehmke u. Baumert 2007, S. 323). Indikator für die soziale Herkunft ist der höchste sozioökonomische Status (HISEI) in der Familie

\begin{tabular}{|c|c|c|c|c|c|c|c|c|c|c|c|c|}
\hline \multirow[t]{2}{*}{ Staat } & \multicolumn{4}{|c|}{ PISA 2000} & \multicolumn{4}{|c|}{ PISA 2003} & \multicolumn{4}{|c|}{ PISA 2006} \\
\hline & b & $(S E)$ & $R^{2}$ & $(S E)$ & b & $(S E)$ & $R^{2}$ & $(S E)$ & $b$ & $(S E)$ & $R^{2}$ & $(S E)$ \\
\hline Däner & 29 & $(1,9)$ & 9,3 & $(1,2)$ & 25 & $(1,7)$ & 7,1 & $(0,9)$ & 23 & $(1,8)$ & 7,8 & $(1,4)$ \\
\hline Finnl & 21 & $(1,8)$ & 5,5 & $(0,8)$ & 18 & $(1,3)$ & 5,2 & $(0,7)$ & 18 & $(1,3)$ & 5,6 & $(0,9)$ \\
\hline Islan & 19 & $(1,4)$ & 4,7 & $(0,7)$ & 12 & $(1,8)$ & 1,5 & $(0,5)$ & 18 & $(1,6)$ & 3,9 & $(0,9)$ \\
\hline Norw & 30 & $(2,0)$ & 7,6 & $(1,0)$ & 29 & $(1,9)$ & 7,3 & $(0,9)$ & 31 & $(2,0)$ & 8,4 & $(1,1)$ \\
\hline Schwe & 27 & $(1,5)$ & 8,8 & $(1,0)$ & 26 & $(1,8)$ & 7,6 & $(0,9)$ & 28 & $(1,9)$ & 8,1 & $(0,9)$ \\
\hline Deutschland & 45 & $(2,1)$ & 15,8 & $(1,5)$ & 38 & $(2,0)$ & 14,0 & $(1,3)$ & 35 & $(2,0)$ & 10,6 & $(1,6)$ \\
\hline $\begin{array}{l}\text { OECD- } \\
\text { Durchschnitt }\end{array}$ & 30 & $(0,4)$ & 10,5 & $(0,3)$ & 28 & $(0,4)$ & 9,3 & $(0,2)$ & 30 & $(0,7)$ & 9,5 & $(0,6)$ \\
\hline
\end{tabular}


sozialen Herkunft mit der Kompetenz der Jugendlichen unterscheiden sich die nordischen Länder vom OECD-Durchschnitt aber insbesondere von Deutschland. In den nordischen Staaten ist diese Kopplung vergleichsweise gering, was in Deutschland gerade im Jahr 2000 nicht der Fall war. Anzumerken ist jedoch, dass in Deutschland im Gegensatz zu den nordischen Staaten signifikante positive Veränderungen, d.h. eine Entkopplung des Zusammenhangs, zu verzeichnen sind (Ehmke u. Baumert 2007; vgl. auch OECD 2007b, S. 131). Insgesamt lässt sich jedoch folgern, dass es in den nordischen Ländern im internationalen Vergleich gut gelingt, herkunftsbedingte soziale Disparitäten gering zu halten. Besonders erfolgreich ist hier wiederum Finnland, aber auch in Island ist der Zusammenhang vergleichsweise klein.

\subsubsection{Migrationshintergrund}

Neben der sozialen Herkunft ist auch die ethnische oder kulturelle Herkunft der Jugendlichen ein Aspekt, der bei PISA mit Blick auf die Chancengerechtigkeit von Bildungssystemen untersucht wird. Als Indikator hierfür wird bei PISA der Migrationshintergrund erhoben, da man - unter den Besonderheiten der Migrationssituation in Mittel- und Nordeuropa - einen hohen Anteil an Jugendlichen mit Migrationshintergrund als besondere Herausforderung für ein Bildungssystem begreifen kann (und als Nachteil bei internationalen Leistungsvergleichen). Von Migrationshintergrund wird gesprochen, wenn die untersuchten Jugendlichen oder ihre beiden Elternteile nicht in dem Land geboren sind, in dem sie zum Zeitpunkt der Datenerhebung leben (OECD 2009).

Unterschiede ergeben sich zwischen den nordischen Ländern schon im Hinblick auf den Anteil von Jugendlichen mit Migrationshintergrund an der Gruppe der Fünfzehnjährigen. Dieser Anteil ist nur in Dänemark und Schweden ausreichend groß, um die Kompetenzwerte der beiden Gruppen miteinander zu vergleichen. Dasselbe gilt für Deutschland, wobei der Anteil von Dänemark (ca. 7\%) am geringsten ist, gefolgt von Schweden (ca. $11 \%$ ), in Deutschland ist er im Vergleich dieser drei Staaten am höchsten (ca. 15\%) (Walter u. Taskinen 2007).

Der Vergleich der Kompetenzwerte von Jugendlichen mit und ohne Migrationshintergrund zeigt, dass Jugendliche mit Migrationshintergrund in allen drei Ländern im Durchschnitt geringere naturwissenschaftliche Kompetenzen erreichen als Jugendliche ohne Migrationshintergrund. In den nordischen Ländern, in deren Bildungssystemen ähnliche Anteile von Jugendlichen mit Migrationshintergrund wie in Deutschland beschult werden, gelingt es damit ebenso wie in Deutschland nicht ausreichend, diese Jugendlichen mit denselben Kompetenzen auszustatten wie die Jugendlichen ohne Migrationshintergrund (Walter u. Taskinen 2007).

\subsubsection{Geschlecht}

Wie sieht es nun mit Disparitäten nach Geschlecht aus? Gelingt es in den nordischen Staaten in allen Kompetenzbereichen zwischen den Geschlechtern vergleichbare Kompetenzniveaus zu entwickeln? Und wie fallen Geschlechterdifferenzen im Bereich von Emotionen und Motivation aus, etwa wenn man das fachspezifische Selbstkonzept der Jugendlichen betrachtet. 
In allen drei PISA-Erhebungen hat sich durchgängig gezeigt, dass insbesondere in der Lesekompetenz deutliche Kompetenzunterschiede zwischen Jungen und Mädchen bestehen. Die nordischen Länder bilden in dieser Hinsicht keine Ausnahme. Auch hier sind die Mädchen den Jungen in der Lesekompetenz deutlich überlegen. In Finnland beträgt die Differenz sogar bis zu 50 Punkte auf der PISA-Skala (OECD 2001, 2004, 2007a). Für Deutschland liegt diese Differenz bei etwa 40 Punkten. Im Durchschnitt aller OECD-Staaten erzielen die Jungen in der Mathematik und in den Naturwissenschaften bedeutend höhere Kompetenzwerte als die Mädchen. Dies gilt für die nordischen Staaten nicht im gleichen Maße. Teilweise zeigt sich in den nordischen Ländern sogar eine Überlegenheit der Mädchen gegenüber den Jungen (OECD 2004). Insgesamt sind die Unterschiede zwischen den Jungen und den Mädchen in der Mathematik und in den Naturwissenschaften gering und häufig nicht statistisch bedeutsam. Auch in Deutschland bestehen keine bedeutsamen Unterschiede in der naturwissenschaftlichen Kompetenz von Jungen und Mädchen. In der Mathematik hingegen sind die Jungen den Mädchen signifikant überlegen, wenn auch nicht im selben Ausmaß wie in der Lesekompetenz (OECD 2001; Blum et al. 2004; Frey et al. 2007; Rost et al. 2004; Prenzel et al. 2007b). Damit weisen die Befunde darauf hin, dass es in den nordischen Ländern in den Bereichen Mathematik und Naturwissenschaften weitgehend gelingt, Kompetenzunterschiede zwischen Jungen und Mädchen gering zu halten oder gar zu vermeiden. In Deutschland gilt dies nur für die Naturwissenschaften.

Entscheidend für die aktive Teilhabe an einer Wissensgesellschaft sind jedoch nicht nur die kognitiven Kompetenzen der Jugendlichen, sondern auch ihre Emotionen und Motivationen, beispielsweise ihr fachspezifisches Fähigkeitsselbstkonzept, das für die spätere Studien- und Berufswahl eine wichtige Rolle spielen kann. Vor dem Hintergrund der Tatsache, dass insbesondere Mädchen wenig Interesse an naturwissenschaftlichen und technischen Berufen zeigen, sollen deshalb auch die Geschlechterdifferenzen im Hinblick auf das naturwissenschaftliche Fähigkeitsselbstkonzept betrachtet werden. In PISA 2006 wurde ebensolches als eine über alle naturwissenschaftlichen Fächer generalisierte Einschätzung der eigenen Kompetenz erhoben (Schütte et al. 2007).

Für das naturwissenschaftliche Fähigkeitsselbstkonzept ergeben sich in allen nordischen Ländern mit Ausnahme von Finnland und Norwegen Unterschiede zwischen Jungen und Mädchen mit Effektstärken, die bei circa 0,2 liegen. Hierbei weisen die Jungen durchweg positivere Fähigkeitsselbstkonzepte als die Mädchen auf (Schütte et al. 2007). In Deutschland beträgt die Effektstärke der Geschlechterdifferenzen 0,15. Damit sind Geschlechterdifferenzen für das naturwissenschaftliche Fähigkeitsselbstkonzept in Finnland, Norwegen und Deutschland belegt, aber von der Größenordnung her als wenig dramatisch einzuschätzen. In Dänemark, Island und Schweden sind die Effektgrößen etwas höher, aber immer noch als eingeschränkt bedeutungsvoll zu bewerten. Damit scheint es in den nordischen Staaten und auch in Deutschland in den Naturwissenschaften sowohl im Hinblick auf die Kompetenz als auch im Hinblick auf das fachspezifische Fähigkeitsselbstkonzept nur geringe Unterschiede zwischen den Geschlechtern zu geben. 


\subsection{Bedingungsfaktoren auf der Ebene von Schule und Unterricht}

Das vorangegangene Kapitel hat gezeigt, wie gut es den nordischen Staaten im internationalen Vergleich und im Vergleich mit Deutschland gelingt, soziale Disparitäten auszugleichen. Dennoch lassen sich diese nie ganz ausschalten. Bedingungsfaktoren für die Bildungsergebnisse von Schülerinnen und Schülern, die sich verändern lassen, finden sich jedoch auf der Ebene von Schule und Unterricht (vgl. auch Carlgren 2009). Deswegen beleuchten wir im folgenden Kapitel solche Bedingungsfaktoren, um zu beschreiben, inwiefern sich die nordischen Staaten im internationalen Vergleich abheben, um daraus eventuell Hinweise für eine kompetenzförderliche Gestaltung von Schule und Unterricht abzuleiten.

\subsubsection{Klassengröße und Unterrichtszeit}

Die Schule ist der Ort, an dem alle Schülerinnen und Schüler gleichermaßen die Chance haben, sich mit relevanten Inhalten auseinanderzusetzen und Kompetenzen aufzubauen (OECD, in Vorbereitung). In Anbetracht der verschiedenartigen Organisation von Schulsystemen in Kombination mit der Unterschiedlichkeit von Schulen ist jedoch anzunehmen, dass Schülerinnen und Schüler in ihren Schulen mit unterschiedlichen Bedingungen für das Lernen konfrontiert werden. Dieses Kapitel betrachtet zwei dieser Bedingungen: die Klassengröße und die Unterrichtszeit.

Die Klassengröße wird in der empirischen Forschung immer wieder als Bedingungsfaktor berücksichtigt, der für den Lernerfolg von Schülerinnen und Schülern eine Rolle spielen könnte, wenngleich die Annahmen selten durch Evidenz gestützt werden (vgl. z. B. Achilles 1999; Finn u. Achilles 1990, 1999; Lankes u. Carstensen 2007). Bei PISA wird die Klassengröße im Rahmen des Fragebogens erfasst, der von den Schulleitungen der teilnehmenden Schulen ausgefüllt wird. Im Rahmen von PISA 2006 wurden keine Klassengrößen berichtet. In den beiden vorangegangenen Erhebungsrunden wurde jeweils die Klassengröße in dem Fach erhoben, welches im Fokus der Erhebung stand (im Jahr 2000 Unterricht in der Testsprache; im Jahr 2003 Mathematik). Im OECD-Durchschnitt betrug die Klassengröße im Unterricht in der Testsprache 24,6 (Schümer 2001, S. 422) und im Unterricht in Mathematik 23,3 (Senkbeil u. Drechsel 2004, S. 290). Für beide Fächer liegt die Klassengröße in allen nordischen Staaten unterhalb des OECD-Durchschnitts. Hierbei werden aus Dänemark die vergleichsweise kleinsten Klassen berichtet. Die Klassengröße in Deutschland liegt etwas höher als in den nordischen Staaten und damit im OECD-Durchschnitt. Die nordischen Staaten heben sich also nur leicht vom internationalen Durchschnitt ab.

Eine weitere wichtige Bedingung für den Aufbau von Kompetenzen in der Schule ist die Zeit, die Schülerinnen und Schüler im Unterricht verbringen (Seidel et al. 2007). In Tab. 3 ist der prozentuale Anteil von Schülerinnen und Schülern dargestellt, die von weniger als zwei beziehungsweise von vier oder mehr Unterrichtsstunden pro Woche in der Testsprache, in Mathematik und in den Naturwissenschaften berichten. Die Angaben sind dem Schülerfragebogen der PISA 2006 Datenerhebung entnommen.

Der Blick auf die Unterrichtszeiten in den nordischen Ländern zeigt besonders in den Naturwissenschaften Abweichungen vom OECD-Durchschnitt. In den nordischen Staa- 
Tab. 3: Wöchentliche Lernzeit in den Naturwissenschaften in den nordischen Staaten, in Deutschland und im OECD-Durchschnitt: Prozentuale Anteile von Schülerinnen und Schülern, die von weniger als zwei beziehungsweise von vier oder mehr Unterrichtsstunden berichten

\begin{tabular}{|c|c|c|c|c|c|c|c|c|c|c|c|c|}
\hline \multirow{3}{*}{$\begin{array}{l}\begin{array}{l}\text { Unter- } \\
\text { richtszeit }\end{array} \\
\text { Staat }\end{array}$} & \multicolumn{4}{|c|}{ Naturwissenschaften } & \multicolumn{4}{|c|}{ Mathematik } & \multicolumn{4}{|c|}{ Testsprache } \\
\hline & \multicolumn{2}{|c|}{$\begin{array}{l}\text { Weniger als } \\
2 \text { Stunden }\end{array}$} & \multicolumn{2}{|c|}{$\begin{array}{l}4 \text { oder mehr } \\
\text { Stunden }\end{array}$} & \multicolumn{2}{|c|}{$\begin{array}{l}\text { Weniger als } \\
2 \text { Stunden }\end{array}$} & \multicolumn{2}{|c|}{$\begin{array}{l}4 \text { oder mehr } \\
\text { Stunden }\end{array}$} & \multicolumn{2}{|c|}{$\begin{array}{l}\text { Weniger als } \\
2 \text { Stunden }\end{array}$} & \multicolumn{2}{|c|}{$\begin{array}{l}4 \text { oder mehr } \\
\text { Stunden }\end{array}$} \\
\hline & $\%$ & $(S E)$ & $\%$ & $(S E)$ & $\%$ & $(S E)$ & $\%$ & $(S E)$ & $\%$ & $(S E)$ & $\%$ & $(S E)$ \\
\hline Dänemark & 17 & $(1,0)$ & 27 & $(1,0)$ & 4 & $(0,3)$ & 66 & $(1,2)$ & 3 & $(0,3)$ & 86 & $(0,7)$ \\
\hline Finnland & 23 & $(0,8)$ & 27 & $(1,4)$ & 11 & $(0,6)$ & 31 & $(1,6)$ & 15 & $(0,9)$ & 20 & $(1,8)$ \\
\hline Island & 23 & $(0,7)$ & 21 & $(0,7)$ & 5 & $(0,3)$ & 74 & $(0,7)$ & 6 & $(0,4)$ & 67 & $(0,7)$ \\
\hline Norwegen & 25 & $(1,1)$ & 7 & $(0,5)$ & 13 & $(0,6)$ & 31 & $(1,0)$ & 13 & $(0,7)$ & 39 & $(1,3)$ \\
\hline Schweden & 20 & $(0,9)$ & 11 & $(0,6)$ & 12 & $(0,6)$ & 15 & $(0,8)$ & 13 & $(0,9)$ & 17 & $(0,9)$ \\
\hline Deutschland & 35 & $(1,1)$ & 32 & $(1,0)$ & 11 & $(0,8)$ & 49 & $(1,0)$ & 14 & $(0,7)$ & 43 & $(1,0)$ \\
\hline OECD & 33 & $(1,0)$ & 29 & $(1,0)$ & 14 & $(0,7)$ & 48 & $(1,1)$ & 15 & $(0,7)$ & 46 & $(1,0)$ \\
\hline
\end{tabular}

ten ist die Unterrichtszeit in den Naturwissenschaften deutlich homogener verteilt als im OECD-Durchschnitt. Mehr als 50\% der Schülerinnen und Schüler haben zwischen zwei und vier Stunden wöchentlichen Unterricht in den Naturwissenschaften (vgl. auch OECD, in Vorbereitung). In Deutschland beispielsweise besteht deutlich mehr Variation zwischen den Schülerinnen und Schülern hinsichtlich der Zeit, die sie im naturwissenschaftlichen Unterricht verbringen (Kobarg et al. 2008; Seidel et al. 2007).

Für die Mathematik zeigt sich, dass in Dänemark und in Island die deutliche Mehrheit aller Schülerinnen und Schüler vier oder mehr Stunden wöchentlichen Unterricht genießt. In Schweden, Finnland und Norwegen hingegen gibt die Mehrzahl der Schülerinnen und Schüler an, zwischen zwei und vier Stunden wöchentlichen Mathematikunterricht zu haben. Die Befunde für die Unterrichtszeit in der Testsprache gestalten sich ähnlich. Damit heben sich nur Schweden, Finnland und Norwegen leicht vom OECD-Durchschnitt ab, da hier die Mehrheit der Schülerinnen und Schüler weniger als vier Unterrichtsstunden in der Testsprache und in Mathematik haben. Dänemark und Island weisen ebenso wie Deutschland große Ähnlichkeit zum Durchschnitt der OECD-Staaten auf.

\subsubsection{Unterrichtsmuster}

Dass Schülerinnen und Schüler Lernzeit im Unterricht verbringen ist insbesondere in der Mathematik und in den Naturwissenschaften eine notwendige, aber keine hinreichende Bedingung für den Aufbau von Kompetenzen (Seidel et al. 2007). Vor diesem Hintergrund wurden im Rahmen der PISA-2006-Datenerhebung Qualitätsmerkmale des naturwissenschaftlichen Unterrichts aus der Sicht von Schülerinnen und Schülern untersucht (OECD, in Vorbereitung). Im Rahmen dieser Analysen wurden drei verschiedene Muster des naturwissenschaftlichen Unterrichts unterschieden. Zusätzlich wurde der Zusammenhang zwischen diesen Mustern und der naturwissenschaftlichen Kompetenz von Schülerinnen und Schülern untersucht (OECD, in Vorbereitung; Seidel et al. 2007). 
Die Befunde zeigen, dass in den nordischen Staaten die Mehrzahl der Schülerinnen und Schüler einen naturwissenschaftlichen Unterricht genießt, der in einem positiven Zusammenhang mit der naturwissenschaftlichen Kompetenz steht. Ähnliches gilt jedoch auch in der Mehrzahl der OECD-Staaten.

\subsection{Fazit - Bedingungsfaktoren für Bildungsergebnisse in den nordischen Ländern}

Betrachtet man die Bedingungsfaktoren auf der Ebene von Schule und Unterricht, so heben sich die nordischen Staaten nur geringfügig vom Durchschnitt der OECD-Staaten, aber auch von Deutschland ab. Beispielsweise sind die Klassen in den nordischen Ländern etwas kleiner und die Unterrichtszeit in den Naturwissenschaften ist in den nordischen Staaten homogener verteilt als in allen anderen Staaten. Ansonsten werden überwiegend Ähnlichkeiten der nordischen Länder mit den OECD-Staaten, aber auch mit Deutschland deutlich.

Im Hinblick auf die Frage, ob es den nordischen Staaten erfolgreicher gelingt, soziale Disparitäten auszugleichen, ergibt sich ein differenzierteres Bild. So sind Jugendliche mit Migrationshintergrund in den beiden nordischen Staaten, in denen ihre Anzahl mit der in Deutschland vergleichbar ist, gegenüber Jugendlichen ohne Migrationshintergrund ebenso wie in Deutschland benachteiligt. Disparitäten zwischen den Geschlechtern bestehen in den nordischen Staaten, ähnlich wie in allen OECD-Staaten, hauptsächlich in der Lesekompetenz. In der Mathematik und den Naturwissenschaften hingegen ergeben sich in den nordischen Ländern kaum Unterschiede zwischen den Geschlechtern, was insbesondere in der Mathematik nicht in allen OECD-Staaten, darunter auch Deutschland, gelingt.

Besonders erfolgreich sind die nordischen Staaten jedoch im Hinblick auf die Entkoppelung des Zusammenhangs zwischen sozialer Herkunft und Kompetenz von Schülerinnen und Schülern. Zwar verringert sich der Zusammenhang in den meisten nordischen Staaten (mit Ausnahme von Dänemark) nicht, wie es beispielsweise in Deutschland der Fall ist, aber er ist konstant geringer ausgeprägt als im OECD-Durchschnitt und in Deutschland (Ehmke u. Baumert 2007). Insbesondere im Hinblick auf die soziale Herkunft gelingt es den nordischen Staaten damit relativ erfolgreich, Chancengerechtigkeit herzustellen, wie sie ja explizit als Ziel ihrer Bildungssysteme formuliert wird (Lie et al. 2003).

\section{Fazit - Der Mythos der nordischen Bildungssysteme}

Ziel dieses Artikels war es, zu beleuchten, ob der Mythos der erfolgreichen nordischen Bildungssysteme sich anhand empirischer Daten absichern lässt. Hierzu sind Befunde aus drei Erhebungszyklen der internationalen Schulleistungsuntersuchung PISA für die nordischen Staaten vorgestellt worden. Diese Befunde bezogen sich einerseits auf die Bildungsergebnisse der Jugendlichen in den nordischen Staaten andererseits wurden unterschiedliche Bedingungsfaktoren für diese Bildungsergebnisse in den nordischen Ländern diskutiert. 
Die dargestellten PISA-Befunde zeigen, dass die Bildungsergebnisse der nordischen Länder meist im guten Durchschnitt der OECD-Staaten liegen. Überdurchschnittliche Leistungen werden jedoch nur von einzelnen Staaten oder in einzelnen Domänen erbracht. Die herausragenden Ergebnisse der finnischen Jugendlichen heben sich nicht nur vom internationalen Durchschnitt ab, sondern bilden auch in der Gruppe der nordischen Staaten eine Ausnahme. Finnland gehört in allen Domänen und über alle drei Erhebungszyklen hinweg zur Spitzengruppe der OECD-Staaten. Derart erfolgreiche Bildungsergebnisse können in keinem anderen nordischen Staat verzeichnet werden. Außerdem weisen die positiven Befunde auch nur in Finnland eine hohe Stabilität auf. In allen anderen nordischen Ländern zeichnet sich über die Zeit tendenziell eine Abschwächung der Kompetenzen ab. Gründe hierfür lassen sich eventuell auf Reformen in den Bildungssystemen der nordischen Staaten zurückführen. Im Hinblick auf den Erfolg ihrer Bildungssysteme scheint eine idealisierende Betrachtung der nordischen Staaten somit kaum gerechtfertigt. Letztlich kann so eigentlich nur von einem „Mythos Finnland“ gesprochen werden, denn trotz der ähnlichen Bildungssysteme und -landschaften in den fünf Staaten erzielt Finnland deutlich bessere Bildungsergebnisse.

Die Befunde zu Bedingungsfaktoren für Bildungsergebnisse weisen darauf hin, dass die nordischen Staaten größtenteils erfolgreich mit den sozialen Disparitäten umgehen, die Schülerinnen und Schüler in die Schule mitbringen. So ist beispielsweise der Zusammenhang zwischen der sozialen Herkunft von Schülerinnen und Schülern und ihrer Kompetenz vergleichsweise gering. Auch Kompetenzunterschiede zwischen den Geschlechtern bestehen in der Mathematik und in den Naturwissenschaften in den nordischen Ländern kaum. Ebenso gering sind die Geschlechterunterschiede im fachspezifischen Fähigkeitsselbstkonzept. In der Lesekompetenz gestaltet sich die Situation jedoch ähnlich wie in allen OECD-Staaten - die Jungen sind den Mädchen deutlich unterlegen. Auch Jugendliche mit Migrationshintergrund erreichen in den nordischen Staaten, in denen ihr Anteil eine Untersuchung der Unterschiede erlaubt, geringere Kompetenzwerte als Jugendliche ohne Migrationshintergrund.

Stellt man die Frage, welche Bedingungen auf der Ebene von Schule und Unterricht den Ausgleich sozialer Disparitäten eventuell begünstigen, so zeigen sich nur geringfügige Unterschiede zu anderen OECD-Staaten. Die Klassen sind in den nordischen Staaten zwar etwas kleiner und die Unterrichtszeit in den Naturwissenschaften deutlich homogener verteilt, aber als Erklärung für den erfolgreicheren Umgang insbesondere mit Unterschieden in der sozialen Herkunft reicht dies kaum aus.

Diese Befunde weisen zusammenfassend darauf hin, dass eine idealisierende Betrachtung der Bildungssysteme aller nordischen Staaten aufgrund der Bildungsergebnisse, die sie in der internationalen Schulleistungsuntersuchung PISA erzielt haben, kaum gerechtfertigt ist. Allein das finnische Bildungssystem scheint sehr erfolgreich darin zu sein, eine große Zahl der Jugendlichen auf die Teilhabe an einer technologisierten Wissensgesellschaft vorzubereiten. Allerdings fällt es bei einer vergleichenden Betrachtung der nordischen Länder schwer, die Bedingungen zu identifizieren, die den Erfolg des finnischen Bildungssystems auch im Vergleich mit den anderen nordischen Bildungssystemen erklären könnten. Eventuell sind Bedingungen für den Erfolg des finnischen Bildungssystems bei PISA in historischen, sozialen oder kulturellen Faktoren begründet, die in den PISAErhebungen nicht abgebildet werden (Nóvoa u. Yariv-Mashal 2003; Simola 2005). 
Open Access Dieser Artikel unterliegt den Bedingungen der Creative Commons Attribution NoncommercialLicense. Dadurch sind die nichtkommerzielle Nutzung, Verteilung und Reproduktion erlaubt, sofernder/die Originalautor/en und die Quelle angegeben sind.

\section{Literatur}

Achilles, C. M. (1999). Let's put kids first, finally: Getting class size right. Thousand Oaks: Corwin Press.

Aktionsrat Bildung (2007). Bildungsgerechtigkeit. Wiesbaden: VS-Verlag für Sozialwissenschaften.

Baumert, J., Stanat, P., \& Demmrich, A. (2001a). PISA 2000: Untersuchungsgegenstand, theoretische Grundlagen und Durchführung der Studie. In J. Baumert, E. Klieme, M. Neubrand, M. Prenzel, U. Schiefele, W. Schneider et al. (Hrsg.), PISA 2000. Basiskompetenzen von Schülerinnen und Schülern im internationalen Vergleich (S. 15-68). Opladen: Leske + Budrich.

Baumert, J., Klieme, E., Neubrand, M., Prenzel, M., Schiefele, U., Schneider, W. et al. (2001b). PISA 2000. Basiskompetenzen von Schülerinnen und Schülern im internationalen Vergleich. Opladen: Leske + Budrich.

Baumert, J., Lehmann, R., Lehrke, M., Schmitz, B., Clausen, M., Hosenfeld, I., et al. (1997). TIMSS - Mathematisch-naturwissenschaftlicher Unterricht im internationalen Vergleich. Deskriptive Befunde. Opladen: Leske + Budrich.

Beaton, A. E., Martin, M. O., Mullis, I. V. S., Gonzales, E. J., Smith, T. A., \& Kelly, D. L. (1996). Science achievement in the middle school years: IEA's Third International Mathematics and Science Study (TIMSS). Chestnut Hill: TIMSS \& PIRLS international study center, Boston College.

Blum, W., Neubrand, M., Ehmke, T., Senkbeil, M., Jordan, A., Ulfig, F., et al. (2004). Mathematische Kompetenz. In M. Prenzel, J. Baumert, W. Blum, R. Lehmann, D. Leutner, M. Neubrand et al. (Hrsg.), PISA 2003. Der Bildungsstand der Jugendlichen in Deutschland. Ergebnisse des zweiten internationalen Vergleichs (S. 47-92). Münster: Waxmann.

Bundesministerium für Bildung und Forschung. (2003). Vertiefender Vergleich der Schulsysteme ausgewählter PISA-Teilnehmerstaaten. Bonn: BMBF.

Carstensen, C. H., Prenzel, M., \& Baumert, J. (2008). Trendanalysen: Wie haben sich die Kompetenzen in Deutschland zwischen PISA 2000 und PISA 2006 entwickelt? In M. Prenzel \& J. Baumert (Hrsg.), Vertiefende Analysen zu PISA 2006 (Sonderheft 10 der Zeitschrift für Erziehungswissenschaft, S. 11-34). Wiesbaden: VS-Verlag für Sozialwissenschaften.

Carlgren, I. (2009). The Swedish comprehensive school - lost in transition? Zeitschrift für Erziehungswissenschaft, 12 .

Carstensen, C. H., Frey, A., Walter, O., \& Knoll, S. (2007). Technische Grundlagen des dritten internationalen Vergleichs. In M. Prenzel, C. Artelt, J. Baumert, W. Blum, M. Hammann, E. Klieme et al. (Hrsg.), PISA 2006. Die Ergebnisse der dritten internationalen Vergleichsstudie (S. 367-390). Münster: Waxmann.

Drechsel, B., \& Artelt, C. (2007). Lesekompetenz. In M. Prenzel, C. Artelt, J. Baumert, W. Blum, M. Hammann, E. Klieme et al. (Hrsg.), PISA 2006. Die Ergebnisse der dritten internationalen Vergleichsstudie (S. 225-248). Münster: Waxmann.

Ehmke, T., \& Baumert, J. (2007). Soziale Herkunft und Kompetenzerwerb: Vergleiche zwischen PISA 2000, 2003 und 2006. In M. Prenzel, C. Artelt, J. Baumert, W. Blum, M. Hammann, E. Klieme et al. (Hrsg.), PISA 2006. Die Ergebnisse der dritten internationalen Vergleichsstudie (S. 309-336). Münster: Waxmann.

Ehmke, T., \& Siegle, T. (2005). ISEI, ISCED, HOMEPOS, ESCS. Indikatoren der sozialen Herkunft bei der Quantifizierung von sozialen Disparitäten. Zeitschrift für Erziehungswissenschaft, 8, 521-540. 
Finn, J. D., \& Achilles, C. M. (1990). Answers and questions about class size: A state wide experiment. American Educational Research Journal, 27, 557-577.

Finn, J. D., \& Achilles, C. M. (1999). Tennessee's class size study: Findings, implications, misconceptions. Educational Evaluation and Police Analysis, 21(2), 97-109.

Frey, A., Asseburg, R., Carstensen, C. H., Ehmke, T., \& Blum, W. (2007). Mathematische Kompetenz. In M. Prenzel, C. Artelt, J. Baumert, W. Blum, M. Hammann, E. Klieme et al. (Hrsg.), PISA 2006. Die Ergebnisse der dritten internationalen Vergleichsstudie (S. 249-276). Münster: Waxmann.

Hautamäki, J., Harjunen, E., Hautamäki, A., Karjalainen, T., Kupiainen, S., Laaksonen, S., et al. (2008). PISA 2006 Finland. Analyses - reflections - explanations. Helsinki: Finnish ministry of education.

Kahl, R. (2003). Spitze - Schulen am Wendekreis der Pädagogik. Warum Schulen in Skandinavien gelingen (Filmdokumentation). Frankfurt a.M.: GEW.

Kobarg, M., Altmann, U., Wittwer, J., Seidel, T., \& Prenzel, M. (2008). Naturwissenschaftlicher Unterricht im Ländervergleich. In M. Prenzel, C. Artelt, J. Baumert, W. Blum, M. Hammann, E. Klieme et al. (Hrsg.), PISA 2006 in Deutschland. Die Kompetenzen der Jugendlichen im dritten Ländervergleich (S. 265-296). Münster: Waxmann.

Lankes, E. M., \& Carstensen, C. H. (2007). Der Leseunterricht aus der Sicht der Lehrkräfte. In W. Bos, S. Hornberg, K.-H. Arnold, G. Faust, L. Fried, E.-M. Lankes et al. (Hrsg.), IGLU 2006. Lesekompetenzen von Grundschülern in Deutschland im internationalen Vergleich (S. 161-191). Münster: Waxmann.

Lie, S., Linnakylä, P., \& Roe, A. (2003). Northern lights on PISA. Oslo: University of Oslo, Department of Teacher Education and School development.

Matti, T. (Ed.) (2009). Northern lights on PISA 2006. Differences and similiarities in the Nordic countries. Copenhagen: Nordic Council of Ministers.

Nóvoa, A., \& Yariv-Mashal, T. (2003). Comparative research in education: A mode of governance or a historical journey. Comparative Education, 39, 423-438.

OECD (2001). Knowledge and skills for life. First results from PISA 2000. Paris: OECD.

OECD (2004). Learning for tomorrow's world. First results from PISA 2003. Paris: OECD.

OECD (2007a). PISA 2006. Science competencies for tomorrow's world. Vol. 1: Analysis. Paris: OECD.

OECD (2007b). PISA 2006. Vol. 2: Data. Paris: OECD.

OECD (2009). PISA 2006. Technical report. Paris: OECD.

OECD (in Vorbereitung). PISA 2006. Thematic report: Teaching and learning of science. Paris: OECD.

Prenzel, M. (2005). Zur Situation der empirischen Bildungsforschung. In H. Mandl \& B. Koop (Hrsg.), Impulse für die Bildungsforschung. Stand und Perspektiven. Dokumentation eines Expertengesprächs. Deutsche Forschungsgemeinschaft (S. 7-21). Berlin: Akademie Verlag.

Prenzel, M., Artelt, C., Baumert, J., Blum, W., Hamann, M., Klieme, E., et al. (2007a). PISA 2006. Die Ergebnisse der dritten internationalen Vergleichsstudie. Münster: Waxmann.

Prenzel, M., Schöps, K., Rönnebeck, S., Senkbeil, M., Walter, O., Carstensen, C. H., et al. (2007b). Naturwissenschaftliche Kompetenz im internationalen Vergleich. In M. Prenzel, C. Artelt, J. Baumert, W. Blum, M. Hamann, E. Klieme et al. (Hrsg.), PISA 2006. Die Ergebnisse der dritten internationalen Vergleichsstudie (S. 63-105). Münster: Waxmann.

Robitaille, F. (1997). National contexts for mathematics and science education. Vancouver: Pacific Educational Press.

Rost, J., Walter, O., Carstensen, C. H., Senkbeil, M., \& Prenzel, M. (2004). Naturwissenschaftliche Kompetenz. In M. Prenzel, J. Baumert, W. Blum, R. Lehmann, D. Leutner, M. Neubrand et al. (Hrsg.), PISA 2003. Der Bildungsstand der Jugendlichen in Deutschland. Ergebnisse des zweiten internationalen Vergleichs (S. 111-146). Münster: Waxmann. 
Sarjala, J., \& Häkli, E. (2008). Jenseits von PISA. Finnlands Schulsystem und seine neusten Entwicklungen. Berlin: Berliner Wissenschafts-Verlag.

Schümer, G. (2001). Lebens- und Lernbedingungen von Jugendlichen. Institutionelle Bedingungen schulischen Lernens im internationalen Vergleich. In J. Baumert, E. Klieme, M. Neubrand, M. Prenzel, U. Schiefele et al. (Hrsg.), PISA 2000. Basiskompetenzen von Schülerinnen und Schülern im internationalen Vergleich (S. 411-426). Opladen: Leske + Budrich.

Schütte, K., Frenzel, A. C., Asseburg, R., \& Pekrun, R. (2007). Schülermerkmale, naturwissenschaftliche Kompetenz und Berufserwartung. In M. Prenzel, C. Artelt, J. Baumert, W. Blum, M. Hammann, E. Klieme et al. (Hrsg.), PISA 2006. Die Ergebnisse der dritten internationalen Vergleichsstudie (S. 125-146). Münster: Waxmann.

Seidel, T., \& Prenzel, M. (2008). Large scale assessment. In J. Hartig, E. Klieme \& D. Leutner (Eds.), Assessment of competencies in educational contexts (pp. 279-304). Göttingen: Hogrefe \& Huber.

Seidel, T., Prenzel, M., Wittwer, J., \& Schwindt, K. (2007). Unterricht in den Naturwissenschaften. In M. Prenzel, C. Artelt, J. Baumert, W. Blum, M. Hammann, E. Klieme et al. (Hrsg.), PISA 2006. Die Ergebnisse der dritten internationalen Vergleichsstudie (S. 147-180). Münster: Waxmann.

Senkbeil, M., \& Drechsel, B. (2004). Institutionelle und organisatorische Rahmenbedingungen von Schule und Unterricht. In M. Prenzel, J. Baumert, W. Blum, R. Lehmann, D. Leutner, M. Neubrand et al. (Hrsg.), PISA 2003. Der Bildungsstand der Jugendlichen in Deutschland. Ergebnisse des zweiten internationalen Vergleichs (S. 177-190). Münster: Waxmann.

Simola, H. (2005). The Finnish miracle of PISA. Historical and sociological remarks on teaching and teacher education. Comparative Education, 41, 445-470.

Walter, O., \& Taskinen, P. (2007). Kompetenzen und bildungsrelevante Einstellungen von Jugendlichen mit Migrationshintergrund in Deutschland: Ein Vergleich mit ausgewählten OECD Staaten. In M. Prenzel, C. Artelt, J. Baumert, W. Blum, M. Hammann, E. Klieme et al. (Hrsg.), PISA 2006. Die Ergebnisse der dritten internationalen Vergleichsstudie (S. 337-366). Münster: Waxmann. 